\title{
Qualidade de Vida Relacionada à Saúde e Risco de Comorbidade Cardiovascular ao Diagnóstico de Câncer de Mama
}

doi: https://doi.org/10.32635/2176-9745.RBC.2019v65n3.713

\author{
Health-Related Quality of Life and Cardiovascular Comorbidity Risk for Breast Cancer Diagnosis \\ Calidad de Vida Relacionada con la Salud y Riesgo de Comorbilidad Cardiovascular al Diagnóstico de Cáncer de Mama
}

\begin{abstract}
Suzana Sales de Aguiar'; Graziele Marques Rodrigues²; Larissa Nascimento dos Santos ${ }^{3}$; Raphaela Nunes de Lucena; Julia de Melo Ramirez Medina ${ }^{5}$; Karen de Souza Abrahão ${ }^{6}$; Luiz Claudio Santos Thuler7; Ines Echenique Mattos ${ }^{8}$; Anke Bergmann ${ }^{9}$
\end{abstract}

\section{Resumo}

Introduçáo: A literatura sugere que mulheres com doenças cardiovasculares apresentam pior qualidade de vida ao diagnóstico de câncer de mama. Objetivo: Avaliar a associação entre a qualidade de vida relacionada à saúde (QVRS) e as doenças cardiovasculares ao diagnóstico de câncer de mama. Método: Estudo transversal com mulheres diagnosticadas com câncer de mama. A QVRS foi avaliada pelos questionários European Organization for Research and Treatment of Cancer Quality of Life Questionnarie (EORTC QLQ-C30) and Breast Câncer Module (QLQ-BR23) e a comorbidade por meio da Cumulative Illness Rating Scale Geriatric (CIRS-G). Foram calculadas as diferenças entre as médias dos escores de QVRS e comorbidade. A associação foi avaliada por regressão logística múltipla. Resultados: Foram incluídas 953 mulheres com média de idade de 54 anos $(\mathrm{DP} \pm 11,7)$. Apresentavam alguma comorbidade ao diagnóstico de câncer de mama $84,1 \%$ das mulheres. O sistema coração foi afetado em 10,8\% e o sistema vascular em 48,2\%. Após ajuste, observou-se associação entre os escores das funçóes física e sexual e problemas nos sistemas coração e vascular. Foi ainda observada associação entre os piores escores de dor e dispneia e o sistema coraçáo. Em relaçáo à presença de doenças no sistema vascular, este esteve associado à melhor satisfação sexual, melhor perspectiva futura e piores sintomas na mama. Conclusáo: A QVRS se mostrou associada a doenças cardiovasculares em pacientes com câncer de mama em relação à função física, sexual, satisfação sexual, a perspectivas futuras e às escalas de sintomas (dor, dispneia e sintomas na mama).

Palavras-chave: Doenças Cardiovasculares; Neoplasias da Mama; Comorbidade; Qualidade de Vida.

\section{Abstract}

Introduction: The literature suggests that women with cardiovascular diseases have worse quality of life when diagnosed with breast cancer. Objective: To evaluate the association between health-related quality of life (HRQoL) and cardiovascular disease at diagnosis of breast cancer. Method: Cross-sectional study with women diagnosed with breast cancer. HRQoL was assessed by the questionnaires of the European Organization for Research and Treatment of Cancer Quality of Life Questionnarie (EORTC QLQ-C30) and Breast Cancer Module (QLQ-BR23) and comorbidity was assessed by the Cumulative Illness Rating Scale Geriatric (CIRS-G). It were calculated the differences between mean HRQoL values and comorbidity. Logistic multiple regression was used to evaluate the association. Results: 953 women with a mean age of 54 years $(\mathrm{SD} \pm 11.7)$ were included. Comorbidity was presented at diagnosis of breast cancer in $84.1 \%$ of the women. The heart system was affected in $10.8 \%$ and the vascular system in $48.2 \%$. After adjustment, an association between physical and sexual function scores and heart and vascular system problems was observed. An association between worse pain and dyspnea scores and the heart system was also noticed. Regarding the presence of diseases in the vascular system, this was associated with better sexual satisfaction, better future outcome and worse breast symptoms. Conclusion: HRQoL was associated with cardiovascular disease in breast cancer patients, in relation to physical and sexual function, sexual satisfaction, future perspectives and symptom scales (pain, dyspnea and breast symptoms).

Key words: Cardiovascular Diseases; Breast Neoplasms; Comorbidity; Quality of Life.
Resumen

Introducción: La literatura sugiere que las mujeres con enfermedad cardiovascular tienen una peor calidad de vida cuando se les diagnostica cáncer de la mama. Objetivo: Evaluar la asociación entre la calidad de vida relacionada con la salud (CVRS) y la enfermedad cardiovascular en el diagnóstico de cáncer de mama. Método: Estudio transversal con mujeres diagnosticadas con cáncer de mama. La CVRS se evaluó mediante los cuestionarios European Organization for Research and Treatment of Cancer Quality of Life Questionnarie (EORTC QLQ-C30) and Breast Câncer Module (QLQ-BR23) y la comorbilidad se evaluó mediante Cumulative Illness Rating Scale Geriatric (CIRS-G). Se calcularon las diferencias entre las puntuaciones medias de CVRS y la comorbilidad. La asociación se evaluó mediante regresión logística múltiple. Resultados: Se incluyeron 953 mujeres con una edad media de 54 ańos (DP $\pm 11,7)$. Algunas tenían comorbilidad en el diagnóstico de cáncer de mama $84,1 \%$ de las mujeres. El sistema cardíaco se vio afectado en 10,8\% y el sistema vascular en $48,2 \%$. Después del ajuste, se observó una asociación entre las puntuaciones de la función física y sexual y los problemas del corazón y del sistema vascular. También se observó una asociación entre puntajes de dolor y disnea peores y el sistema cardíaco. Con respecto a la presencia de enfermedades en el sistema vascular, esto se asoció con una mejor satisfacción sexual, una mejor perspectiva futura y peores síntomas mamarios. Conclusión: La CVRS se asoció con la enfermedad cardiovascular en pacientes con cáncer de mama, con respecto a la función física, la función sexual, la satisfacción sexual, las perspectivas futuras y las escalas de síntomas (dolor, disnea y síntomas de mama).

Palabras clave: Enfermedades Cardiovasculares; Neoplasias de la Mama; Comorbilidad; Calidad de Vida.

\footnotetext{
${ }^{1}$ Coordenação de Pesquisa Clínica do Instituto Nacional de Câncer José Alencar Gomes da Silva (INCA). Rio de Janeiro (RJ), Brasil. Orcid iD: https://orcid.org/0000-0003-1963-1294 2 Departamento de Epidemiologia e Métodos Quantitativos em Saúde (DEMQS)/Escola Nacional de Saúde Pública Sérgio Arouca/Fundação Oswaldo Cruz (Ensp/ Fiocruz). Rio de Janeiro (RJ), Brasil. Orcid iD: https://orcid.org/0000-0002-4299-2349

${ }^{3}$ Coordenação de Pesquisa Clínica do INCA. Rio de Janeiro (RJ), Brasil. Orcid iD: https://orcid.org/0000-0003-2114-8840

${ }^{4}$ Coordenação de Pesquisa Clínica do INCA. Rio de Janeiro (RJ), Brasil. Orcid iD: https://orcid.org/0000-0003-0093-7453

${ }^{5}$ Coordenação de Pesquisa Clínica do INCA. Rio de Janeiro (RJ), Brasil. Orcid iD: https://orcid.org/0000-0003-0879-0409

${ }^{6}$ DEMQS/Ensp/Fiocruz. Rio de Janeiro (RJ), Brasil. Orcid iD: https://orcid.org/0000-0003-2436-8336x

${ }^{7}$ Coordenação de Pesquisa Clínica do INCA. Rio de Janeiro (RJ), Brasil. Orcid iD: https://orcid.org/0000-0003-2550-6537

${ }^{8}$ DEMQS/Ensp/Fiocruz. Rio de Janeiro (RJ), Brasil. Orcid iD: https://orcid.org/0000-0002-7297-1864

${ }^{9}$ Coordenação de Pesquisa Clínica do INCA. Rio de Janeiro (RJ), Brasil. Orcid iD: https://orcid.org/0000-0002-1972-8777

Endereço para correspondência: Suzana Sales de Aguiar. Rua André Cavalcanti, 37 - Sala 9- Anexo - Centro. Rio de Janeiro (RJ), Brasil. CEP 20231-050. E-mail: saguiar@inca.gov.br
} 


\section{INTRODUÇÃO}

O câncer de mama é o tumor mais incidente entre as mulheres no mundo, sendo responsável por $24,2 \%$ dos casos de câncer ${ }^{1}$. No Brasil, foram estimados, para cada ano do biênio 2018-2019, 59.700 casos de câncer de mama, sendo as Regióes Sudeste e Sul as que apresentam maior incidência ${ }^{2}$.

A idade é um importante fator de risco para o desenvolvimento do câncer de mama; mulheres acima de 50 anos apresentam maior incidência ${ }^{3}$. Com o aumento da expectativa de vida, outras doenças crônicas não transmissíveis (DCNT) relacionadas ao envelhecimento populacional são encontradas. Dessa forma, muitas pacientes ao diagnóstico da doença já possuem algum tipo de comorbidade e/ou alguma DCNT prévia, entre elas, as mais incidentes são as cardiovasculares que, de acordo com a World Health Organization (WHO), são consideradas as principais causas de morte no mundo ${ }^{4}$.

Segundo Patnaik et al. ${ }^{5}$, pacientes com câncer de mama acima de 65 anos apresentam maior risco de óbito por doença cardiovascular do que pelo câncer, mesmo se forem diagnosticadas pela neoplasia em estadiamentos iniciais. Os eventos cardíacos após a terapia antineoplásica estão bem documentados na literatura científica ${ }^{6-8}$, assim como os óbitos cardiovasculares associados tanto ao diagnóstico como ao tratamento do câncer de mama?.

Durante todas as fases do tratamento, desde o momento do diagnóstico, as pacientes passam por experiências emocionais e físicas que impactam diretamente na qualidade de vida relacionada à saúde $(\mathrm{QVRS})^{10,11}$. Estudos realizados com aquelas que apresentam comorbidades relatam pior pontuação na escala de qualidade de vida ao diagnóstico de câncer de mama ${ }^{12-14}$.

Portanto, ao diagnóstico de câncer de mama, as mulheres que já apresentam comorbidades parecem também apresentar pior QVRS. Nesse contexto, este estudo tem como objetivo avaliar a associação entre a QVRS e as alteraçóes cardiovasculares ao diagnóstico de câncer de mama.

\section{MÉTODO}

Foi realizado um estudo transversal com mulheres matriculadas no Hospital do Câncer III do Instituto Nacional de Câncer José Alencar Gomes da Silva (HC III/INCA) com diagnóstico de câncer de mama.

Foram incluídas mulheres com idade $\geq 18$ anos, diagnosticadas com câncer de mama, de acordo com a $3^{a}$ edição da Classificação Internacional de Doenças para Oncologia (CID-O) $(\mathrm{C} 50)^{15}$, com indicação de tratamento curativo, no período de abril de 2016 a outubro de 2018. Foram excluídas mulheres que utilizavam dispositivo auxiliar de marcha, com histórico prévio de câncer, sem condiçóes clínicas, psicológicas ou emocionais de responderem ao questionário e que não concordaram em assinar o Termo de Consentimento Livre e Esclarecido (TCLE).

As pacientes foram submetidas à entrevista previamente ao início do primeiro tratamento oncológico. As informaçôes sobre comorbidades e exames médicos foram obtidas por meio de busca direta aos prontuários eletrônico e/ou físico.

A QVRS foi avaliada pela $3^{\text {a }}$ versão do questionário European Organization for Research and Treatment of Cancer Quality of Life Questionnarie (EORTC QLQ-C30), que possui 30 perguntas divididas por escalas, sendo cinco funcionais (funções física, cognitiva, emocional, social e geral) e três de sintomas (fadiga, dor, náuseas e vômito), além de uma escala que avalia a qualidade de vida global e a saúde geral. O módulo específico sobre câncer de mama, em inglês Breast Câncer Module (QLQ-BR23), possui 23 perguntas divididas em duas dimensões, sendo elas a escala funcional (imagem corporal, função sexual, satisfação sexual e perspectivas futuras) e a escala de sintomas (efeitos colaterais da terapia sistêmica, sintomas relacionados ao braço, sintomas relacionados à mama e preocupação com a queda de cabelo). Ambos os questionários foram traduzidos e validados para a população brasileira ${ }^{16}$.

Os desfechos sistemas coração e vascular foram obtidos por meio da Cumulative Illness Rating Scale Geriatric (CIRS-G) ${ }^{17}$, que avaliou a presença e a gravidade das doenças prévias ao diagnóstico em diferentes órgãos e sistemas por meio de um escore de gravidade. A CIRS-G é uma adaptação da escala direcionada para adultos denominada $\mathrm{CIRS}^{18}$ com a inclusão de alguns agravos relacionados aos idosos tornando-se assim mais completa. Além disso, a CIRS-G é bastante usada na oncogeriatria $^{19-22}$. Não existe estudo de validação da CIRS ou CIRS-G para a população brasileira, uma vez que as doenças, contempladas em cada sistema orgânico, estão na $10^{a}$ edição da Classificação Estatística Internacional de Doenças e Problemas Relacionados com a Saúde (CID10) ${ }^{23}$ e são de uso universal.

Foram ainda coletadas informaçóes sociodemográficas (idade ao diagnóstico, renda, raça/cor da pele, estado conjugal, escolaridade, ocupação ao diagnóstico e consumo de álcool e tabaco ao diagnóstico) e clínicas (estádio clínico, tipo histológico, status menopausal, índice de massa corporal e nível de atividade física).

Realizou-se análise descritiva da população por meio de média e desvio-padrão para as variáveis contínuas e determinação de distribuição de frequência para as variáveis categóricas. Para avaliar a associação entre QVRS e comorbidade cardiovascular, foram calculadas as diferenças entre as médias dos escores obtidos e testadas por análise de variância, considerando valor de $\mathrm{p}<0,20$. Regressão logística múltipla foi realizada pelo método enter (stepwise forward), considerando o intervalo de confiança 
de $95 \%$. Todas as análises foram realizadas utilizando o pacote estatístico SPSS, versão 23.0 (IBM).

Esta pesquisa foi submetida ao Comitê de Ética em Pesquisa do INCA sob parecer n. ${ }^{\circ} 1.400 .320$, conforme a resolução CNS n. ${ }^{\circ} 466 / 12$, que dispóe sobre as Diretrizes e Normas Regulamentadoras de Pesquisas envolvendo seres humanos.

\section{RESULTADOS}

Foram incluídas 953 mulheres com diagnóstico de câncer de mama no período do estudo. A média de idade foi de 54 anos $(\mathrm{DP} \pm 11,7)$, sendo a maioria com raça/cor de pele náo branca $(65,0 \%)$, com mais de oito anos de estudo $(68,8 \%)$ e com renda superior a um salário mínimo $(82,9 \%)$ (Tabela 1$)$.

Tabela 1. Características sociodemográficas da população do estudo $(\mathrm{n}=953)$

\begin{tabular}{|c|c|c|}
\hline Variáveis & $\mathbf{N}$ & $\%$ \\
\hline \multicolumn{3}{|l|}{ Idade } \\
\hline Média ( $\pm D P)$ & $54,05(11,72)$ & \\
\hline \multicolumn{3}{|c|}{ Renda em salários mínimos** } \\
\hline Média ( $\pm \mathrm{DP})$ & $2,65(2,65)$ & \\
\hline \multicolumn{3}{|l|}{ Idade } \\
\hline$<50$ anos & 343 & 36,0 \\
\hline$\geq 50$ anos & 610 & 64,0 \\
\hline \multicolumn{3}{|l|}{ Raça/cor da pele } \\
\hline Branca & 334 & 35,0 \\
\hline Não branca* & 619 & 65,0 \\
\hline \multicolumn{3}{|l|}{ Estado conjugal } \\
\hline Com companheiro & 480 & 50,4 \\
\hline Sem companheiro & 473 & 49,6 \\
\hline \multicolumn{3}{|c|}{ Escolaridade (anos de estudo) } \\
\hline$<8$ anos & 297 & 31,2 \\
\hline$\geq 8$ anos & 656 & 68,8 \\
\hline \multicolumn{3}{|l|}{ Renda** } \\
\hline < 1 salário mínimo & 152 & 15,9 \\
\hline$\geq 1$ salário mínimo & 790 & 82,9 \\
\hline Sem informação & 11 & 1,2 \\
\hline \multicolumn{3}{|c|}{ Ocupação ao diagnóstico } \\
\hline Trabalhava & 593 & 62,2 \\
\hline Não trabalhava & 360 & 37,8 \\
\hline \multicolumn{3}{|c|}{ Consumo de álcool nos últimos 30 dias } \\
\hline Sim & 247 & 25,9 \\
\hline Não & 703 & 73,8 \\
\hline Sem informação & 3 & 0,3 \\
\hline \multicolumn{3}{|c|}{ Consumo de tabaco ao diagnóstico } \\
\hline Não fuma & 645 & 67,7 \\
\hline Ex-tabagista & 221 & 23,2 \\
\hline Tabagista atual & 85 & 8,9 \\
\hline Sem informação & 2 & 0,2 \\
\hline
\end{tabular}

Em relação às características clínicas, a maior frequência foi de diagnósticos em estádio avançado (54,5\%), com tipo histológico carcinoma ductal infiltrante $(83,8 \%)$. As mulheres estavam na pós-menopausa em $62,7 \%$ dos casos e, em sua maioria, apresentavam sobrepeso $(36,8 \%)$ e obesidade $(35,9 \%)$ (Tabela 2$)$.

Tabela 2. Características clínicas e comorbidades da população do estudo $(\mathrm{n}=953)$

\begin{tabular}{|c|c|c|}
\hline Variáveis & $\mathbf{N}$ & $\%$ \\
\hline \multicolumn{3}{|l|}{ Estádio clínico } \\
\hline$<I I B$ & 418 & 43,9 \\
\hline$\geq \mathrm{IIB}$ & 520 & 54,5 \\
\hline Sem informação & 15 & 1,6 \\
\hline \multicolumn{3}{|l|}{ Tipo histológico do tumor } \\
\hline CDI & 798 & 83,8 \\
\hline Outros & 148 & 15,5 \\
\hline Sem informação & 7 & 0,7 \\
\hline \multicolumn{3}{|l|}{ Status menopausal } \\
\hline Pós-menopausa & 597 & 62,7 \\
\hline Pré-menopausa & 329 & 34,5 \\
\hline Sem informação & 27 & 2,8 \\
\hline \multicolumn{3}{|l|}{ Índice de massa corporal } \\
\hline Baixo peso & 11 & 1,2 \\
\hline Eutrófico & 219 & 23,0 \\
\hline Sobrepeso & 351 & 36,8 \\
\hline Obesidade & 342 & 35,9 \\
\hline Sem informação & 30 & 3,1 \\
\hline \multicolumn{3}{|l|}{ Escore CIRS-G categórica } \\
\hline Sem comorbidade & 152 & 15,9 \\
\hline Comorbidade leve & 284 & 29,8 \\
\hline Comorbidade moderada & 485 & 50,9 \\
\hline Comorbidade grave & 32 & 3,4 \\
\hline \multicolumn{3}{|l|}{ Sistema coração afetado } \\
\hline Sim & 103 & 10,8 \\
\hline Não & 850 & 89,2 \\
\hline \multicolumn{3}{|l|}{ Sistema vascular afetado } \\
\hline Sim* & 459 & 48,2 \\
\hline Não & 494 & 51,8 \\
\hline
\end{tabular}

Ao avaliar as comorbidades ao diagnóstico de câncer de mama pela escala CIRS-G, $84,1 \%$ das mulheres apresentavam alguma comorbidade, sendo $29,8 \%$ classificadas como leve, $50,9 \%$ moderada e $3,4 \%$ grave. O sistema coração e vascular foram afetados em $10,8 \%$ e $48,2 \%$, respectivamente (Tabela 2). As principais doenças encontradas no sistema coração foram disfunção diastólica grau $1(7,03 \%)$; insuficiência mitral $(1,36 \%)$ e arritmia $(0,95 \%)$; e, no sistema vascular, hipertensão arterial $(46,1 \%)$, dislipidemia $(2,83 \%)$ e varizes $(1,78 \%)$.

Ao diagnóstico de câncer de mama, maiores escores foram observados nas escalas de função (EORTC C-30) 
para função física e social. Verificou-se que as mulheres com problemas nos sistemas coração $(\mathrm{p}=0,008)$ e vascular $(\mathrm{p}<0,001)$ apresentaram pior escore na funçấo física. Em relaçáo à escala de sintomas, piores escores foram observados para insônia e dor. Aquelas com problemas no sistema coração apresentaram piores escores nos sintomas dor $(\mathrm{p}=0,031)$ e dispneia $(\mathrm{p}=0,007)$ (Tabela 3$)$.

Ao avaliar a QVRS pelo EORTC BR-23, foi observada pior função no domínio função sexual e pior sintoma no braço. Ao comparar os escores de acordo com as comorbidades, observou-se pior função sexual entre aquelas com problemas nos sistemas coração $(\mathrm{p}=0,001)$ e vascular $(\mathrm{p}<0,001)$. Além disso, aquelas com problemas no sistema vascular também apresentaram pior escore na satisfação sexual $(\mathrm{p}=0,026)$ e melhor escore na perspectiva futura ( $\mathrm{p}=0,037)$ e queda de cabelo $(\mathrm{p}=0,005)$ (Tabela 4).

Após ajuste, ao avaliar as associações entre os escores das escalas de função da QVRS pelo EORTC QLQ C-30 e comorbidade ao diagnóstico de câncer de mama, a cada aumento de um ponto no escore da função física, foi observada diminuição de $1,1 \%$ no risco de problemas no sistema coraçáo (OR=0,989 IC 95\% 0,979 a 0,999; $\mathrm{p}=0,025)$ e de $1,4 \%$ no risco de problemas no sistema vascular $(\mathrm{OR}=0,986$ IC 95\% 0,986 a 0,994; $\mathrm{p}<0,001)$. Nas escalas do EORTC QLQ BR-23, a cada aumento de um ponto no escore da função sexual, houve diminuição

Tabela 3. Qualidade de vida relacionada à saúde (EORTC QLQ C-30) e comorbidades ao diagnóstico de câncer de mama ( $\mathrm{n=953)}$

\begin{tabular}{|c|c|c|c|c|c|c|c|}
\hline \multirow{3}{*}{ EORTC QLQ C-30 } & \multirow{3}{*}{ Total } & \multicolumn{3}{|c|}{ Problema no sistema coração } & \multicolumn{3}{|c|}{ Problema no sistema vascular } \\
\hline & & Não & Sim & & Não & Sim & \\
\hline & & \multicolumn{3}{|c|}{$850(89,2 \%) 103(10,8 \%)$} & $494(51,8)$ & $459(48,2)$ & \\
\hline Escala de função* & Média (DP) & Média (DP) & Média (DP) & p valor & Média (DP) & Média (DP) & p valor \\
\hline Qualidade de vida global & $69,5(23,3)$ & $69,9(23,1)$ & $65,6(24,7)$ & 0,074 & $69,7(22,5)$ & $69,2(24,1)$ & 0,729 \\
\hline Função física & $83,0(19,8)$ & $83,6(19,6)$ & $78,0(21,0)$ & 0,008 & $85,6(19,1)$ & $80,2(20,3)$ & $<0,001$ \\
\hline Função geral & $78,7(30,3)$ & $79,2(30,0)$ & $74,5(32,9)$ & 0,144 & $80,4(28,7)$ & $76,8(32,0)$ & 0,068 \\
\hline Função cognitiva & $74,4(28,8)$ & $74,2(29,1)$ & $75,9(26,3)$ & 0,570 & $75,6(29,0)$ & $73,1(28,4)$ & 0,186 \\
\hline Função emocional & $56,1(31,4)$ & $55,8(31,5)$ & $59,0(30,8)$ & 0,330 & $55,8(31,0)$ & $56,5(31,8)$ & 0,713 \\
\hline Função social & $81,1(29,7)$ & $81,4(29,8)$ & $78,4(29,1$ & 0,340 & $80,3(29,7)$ & $81,9(29,7)$ & 0,388 \\
\hline Escala de sintomas** & Média (DP) & Média (DP) & Média (DP) & p valor & Média (DP) & Média (DP) & p valor \\
\hline Fadiga & $22,6(26,1)$ & $22,6(26,1)$ & $23,1(25,7)$ & 0,842 & $22,5(26,5)$ & $22,7(25,7)$ & 0,888 \\
\hline Dor & $31,3(32,8)$ & $30,5(32,8)$ & $37,9(32,1)$ & 0,031 & $30,0(32,0)$ & $32,6(33,6)$ & 0,225 \\
\hline Dispneia & $11,4(24,8)$ & $10,6(23,9)$ & $17,6(30,3)$ & 0,007 & $10,8(24,2)$ & $12,0(25,4)$ & 0,460 \\
\hline Insônia & $37,4(41,8)$ & $37,4(41,9)$ & $37,0(41,2)$ & 0,932 & $36,9(41,4)$ & $37,9(42,3)$ & 0,724 \\
\hline Falta de apetite & $14,2(29,1)$ & $14,1(28,7)$ & $14,7(31,7)$ & 0,841 & $12,7(26,8)$ & $15,7(31,3)$ & 0,111 \\
\hline Náuseas e vômito & $7,3(15,9)$ & $7,0(15,3)$ & $9,9(19,9)$ & 0,077 & $7,1(14,9)$ & $7,5(16,8)$ & 0,723 \\
\hline Constipação intestinal & $18,7(32,7)$ & $18,7(32,8)$ & $18,9(31,9)$ & 0,938 & $18,9(33,0)$ & $18,5(32,4)$ & 0,834 \\
\hline Diarreia & $6,7(19,3)$ & $6,4(18,7)$ & $9,1(23,5)$ & 0,174 & $6,2(18,2)$ & $7,2(20,4)$ & 0,432 \\
\hline Dificuldade financeira & $28,7(39,9)$ & $28,6(39,9)$ & $30,1(39,7)$ & 0,721 & $28,3(39,6)$ & $29,2(40,3)$ & 0,742 \\
\hline
\end{tabular}

Legendas: *Escores mais altos, melhor QVRS; ${ }^{* *}$ Escores mais altos, pior QVRS; DP=Desvio-padráo.

Nota: Os valores estatisticamente significantes estão destacados em negrito.

Tabela 4. Qualidade de vida relacionada à saúde (EORTC QLQ BR-23) e comorbidades ao diagnóstico de câncer de mama ( $\mathrm{n}=953$ )

\begin{tabular}{lcccccccc}
\hline \multirow{2}{*}{ EORTC BR-23 } & \multirow{2}{*}{ Total } & \multicolumn{2}{c}{ Problema no sistema coração } & \multicolumn{2}{c}{ Problema no sistema vascular } \\
\cline { 3 - 8 } & & Náo & Sim & P valor & Não & Sim & P valor \\
\hline Escala de função* & Média (DP) & Média (DP) & Média (DP) & P valor & Média (DP) & Média (DP) & P valor \\
\hline Imagem corporal & $83,1(24,9)$ & $82,8(25,3)$ & $85,9(21,0)$ & 0,244 & $83,1(24,9)$ & $83,2(24,9)$ & 0,946 \\
Função sexual & $33,1(31,5)$ & $34,3(31,8)$ & $22,7(27,3)$ & 0,001 & $40,3(31,9)$ & $25,4(29,2)$ & $<\mathbf{0 , 0 0 1}$ \\
Satisfação sexual & $71,8(29,1)$ & $72,3(29,0)$ & $64,5(29,7)$ & 0,149 & $74,1(27,8)$ & $67,8(30,8)$ & $\mathbf{0 , 0 2 6}$ \\
Perspectiva futura & $35,5(39,1)$ & $36,0(39,2)$ & $31,7(38,5)$ & 0,294 & $33,0(38,0)$ & $38,3(40,2)$ & $\mathbf{0 , 0 3 7}$ \\
\hline Escala de sintomas** & Média (DP) & Média (DP) & Média (DP) & P valor & Média (DP) & Média (DP) & P valor \\
\hline Efeitos da terapia sistêmica & $19,2(17,5)$ & $19,1(17,7)$ & $19,5(16,5)$ & 0,867 & $18,2(16,3)$ & $20,2(18,7)$ & 0,075 \\
Queda de cabelo & $28,8(29,8)$ & $36,2(42,3)$ & $22,7(40,3)$ & 0,155 & $31,4(30,3)$ & $26,0(28,9)$ & $\mathbf{0 , 0 0 5}$ \\
Sintomas na mama & $18,2(24,3)$ & $28,7(29,8)$ & $29,7(29,1)$ & 0,745 & $16,9(24,0)$ & $19,4(24,5)$ & 0,119 \\
Sintomas no braço & $34,9(42,2)$ & $17,8(24,2)$ & $21,2(24,2)$ & 0,177 & $32,7(40,0)$ & $37,2(44,5)$ & 0,429 \\
\hline
\end{tabular}

Legendas: *Escores mais altos, melhor QVRS; ${ }^{* *}$ Escores mais altos, pior QVRS; DP=Desvio-padrão

Nota: Os valores estatisticamente significantes estấo destacados em negrito. 
de $1,2 \%$ no risco de apresentar problemas nos sistemas coração (OR=0,988 IC 95\% 0,980 a 0,996; p=0,003) e vascular $(\mathrm{OR}=0,988$ IC 95\% 0,983 a 0,993; $\mathrm{p}<0,001)$. Foi ainda verificado que, a cada aumento de um ponto no escore de satisfação sexual, houve diminuição de $0,7 \%$ no risco de apresentar problemas no sistema vascular $(\mathrm{OR}=0,993$ IC 95\% 0,986 a 0,999; p=0,030). Em relação ao escore perspectiva futura, um acréscimo de um ponto aumentou em $0,4 \%$ o risco de problemas no sistema vascular $(\mathrm{OR}=1,004 \mathrm{IC} 95 \%$ 1,000 a 1,007; $\mathrm{p}=0,042)$ (Tabela 5).

Ao avaliar as escalas de sintomas do EORTC C-30, a cada acréscimo de um ponto da escala de dor, observou-se aumento de 0,8\% no risco de apresentar problemas no sistema coração $(\mathrm{OR}=1,008$ IC 95\% 1,001 a 1,014; $\mathrm{p}=0,020)$ e de $1 \%$ no risco para escala de dispneia $(\mathrm{OR}=1,010$ IC 95\% 1,002 a 1,017; p=0,009). Na escala de sintomas do QLQ-BR23, a cada aumento de um ponto em sintomas da mama, diminuiu em $0,8 \%$ o risco de problemas no sistema vascular (OR=0,992 IC 95\% 0,988 a 0,997; $\mathrm{p}=0,001$ ) (Tabela 5).

\section{DISCUSSÃO}

Neste estudo, a maioria das mulheres foi diagnosticada em estádio avançado do câncer de mama ( $\geq$ IIB) e com alguma comorbidade, sendo $10,8 \%$ com problemas no sistema coração e 48,2\% no sistema vascular. Observou-se maior risco de doenças do sistema coração nas mulheres com piores funçôes física e sexual, e aquelas com pior sintoma de dor e dispneia. Para o sistema vascular, observou-se maior risco naquelas com piores funçóes física e sexual e pior satisfação sexual. Para sintomas na mama e perspectiva futura, aquelas com melhores escores apresentaram menor risco de problemas no sistema vascular.

O câncer de mama está associado ao aumento de problemas cardiovasculares decorrentes, principalmente, do tratamento oncológico e, quanto mais avançado for o diagnóstico do câncer de mama, mais agressivos serão os tratamentos e, consequentemente, maior o risco de complicaçôes cardiovasculares. Nesse sentido, conhecer a prevalência de doenças cardiovasculares e seus fatores de risco ao diagnóstico de câncer de mama é fundamental para direcionar os tratamentos oncológicos ${ }^{24-26}$.

A prevalência de doenças cardiovasculares ao diagnóstico de câncer de mama varia de acordo com as características da população estudada. Em um estudo com 12.127 mulheres canadenses diagnosticadas com câncer de mama, sendo a maioria (80\%) com idade superior a 50 anos, as comorbidades mais prevalentes ao diagnóstico foram as alterações cardiovasculares (39\%), aferidas pelo índice Rx-Risk-V model ${ }^{27}$. Em outro estudo, realizado na Alemanha, foram incluídas 3.496 mulheres com câncer de mama, em sua maioria em estádio inicial e com idade entre 40 e 79 anos. Após padronização por idade, a prevalência global de doenças coronarianas foi de 3,96\% e de 2,06\% para infarto agudo do miocárdio ${ }^{28}$. Em nossa população, a prevalência de doenças cardíacas foi de 10,8\% e vasculares de $48,2 \%$. Entretanto, os instrumentos utilizados para mensurar as comorbidades foram distintos, assim como as características tumorais das populaçóes estudadas, o que torna difícil comparar essas prevalências.

Em relação à QVRS ao diagnóstico de câncer de mama, em nossa população, foram observados melhores escores para as funçôes física e social e piores sintomas de insônia e dor. Aquelas com piores escores de QVRS apresentaram maior risco de doença nos sistemas coração

Tabela 5. Associação bruta e ajustada entre os escores de QVRS e comorbidade no sistema cardiovascular

\begin{tabular}{|c|c|c|c|c|c|c|c|c|}
\hline \multirow{2}{*}{$\begin{array}{l}\text { EORTC QLQ C-30 } \\
\text { Escala de função }\end{array}$} & \multicolumn{4}{|c|}{ Sistema coração } & \multicolumn{4}{|c|}{ Sistema vascular } \\
\hline & OR bruta & $\mathrm{p}$ valor & OR ajustada & p valor & OR bruta & p valor & OR ajustada & p valor \\
\hline Função física & $0,987(0,978-0,997)$ & 0,008 & $0,989(0,979-0,999)^{a}$ & 0,025 & $0,986(0,980-0,993)$ & $<0,001$ & $0,986(0,978-0,994)^{c}$ & $<0,001$ \\
\hline \multicolumn{9}{|l|}{ Escala de sintomas } \\
\hline Dor & $1,007(1,001-1,013)$ & 0,032 & $1,008(1,001-1,014)^{a}$ & 0,020 & --- & --- & --- & --- \\
\hline Dispneia & $1,009(1,002-1,016)$ & 0,008 & $1,010(1,002-1,017)^{a}$ & 0,009 & --- & --- & --- & --- \\
\hline \multicolumn{9}{|l|}{ EORTC BR-23 } \\
\hline \multicolumn{9}{|l|}{ Escala de função } \\
\hline Função sexual & $0,987(0,980-0,994)$ & 0,001 & $0,988(0,980-0,996)^{b}$ & 0,003 & $0,984(0,980-0,989)$ & $<0,001$ & $0,988(0,983-0,993)^{d}$ & $<0,001$ \\
\hline Satisfação sexual & --- & --- & --- & --- & $0,993(0,986-0,999)$ & 0,027 & $0,993(0,986-0,999)^{\mathrm{e}}$ & 0,030 \\
\hline Perspectiva futura & --- & --- & --- & --- & $1,003(1,000-1,007)$ & 0,038 & $1,004(1,000-1,007)^{f}$ & 0,042 \\
\hline \multicolumn{9}{|l|}{ Escala de sintomas } \\
\hline Sintomas na mama & --- & --- & --- & --- & $0,994(0,989-0,998)$ & $<0,001$ & $0,992(0,988-0,997)^{g}$ & 0,001 \\
\hline
\end{tabular}

Legendas: ajustada por idade e estadiamento clínico; bajustada por estadiamento clínico e ocupação; cajustada por idade, IMC, escolaridade e tabagismo; ${ }^{a}$ ajustada

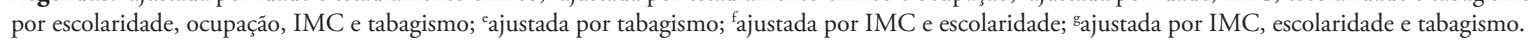


e vascular. Resultados similares foram obtidos em outras populaçóes ${ }^{12,14}$.

Em um estudo de coorte com 542 mulheres dinamarquesas ao diagnóstico de câncer de mama, em sua maioria com estádio clínico I (31\%) ou II (41\%), 10\% apresentavam uma comorbidade e $10 \%$ apresentavam $\geq 2$ comorbidades (aferidas pelo Índice de Comorbidade de Charlson [ICC]). Melhores escores de QVRS (medidas pelo SF-36) foram observados para as funçôes social $(92 \pm 16,6)$ e emocional $(90,7 \pm 17,7)$. As mulheres com uma ou mais comorbidades apresentaram piores escores de QVRS na função física (-4,1 IC $95 \%-6,0$ a -2,2) e na saúde global $(-7,9 \text { IC 95\% - 12,0 a }-3,5)^{12}$.

Em estudo realizado com 339 mulheres espanholas diagnosticadas com câncer de mama, sendo a maioria com estádios I $(44,3 \%)$ e II $(38,1 \%)$, a média do escore de saúde global foi de $69,2( \pm 21,1)$ e melhores escores foram obtidos nas escalas de função física $(92,3 \pm 12,4)$ e função geral $(93,3 \pm 14,3)$, e piores escores nos sintomas de insônia $(31,7 \pm 29,8)$ e fadiga $(15,8 \pm 17,5)$. As mulheres com pior escore de saúde global apresentaram risco 2,07 vezes maior de apresentar comorbidades aferidas pelo ICC $(\mathrm{OR}=2,07 \mathrm{IC} 95 \% 1,29-3,30)^{14}$.

$\mathrm{O}$ artigo apresenta algumas limitações. A inclusão de um número elevado de mulheres pode ter ocasionado a obtençấo de resultados bordelines; este estudo foi realizado com mulheres diagnosticadas com câncer de mama em uma única instituição pública de referência no tratamento oncológico e, por isso, alguns cuidados devem ser considerados na generalização dos seus resultados (validade externa). Além disso, não foi possível a comparação dos nossos resultados com outros estudos, pois os instrumentos utilizados na obtenção dos escores de QVRS e de comorbidades cardiovasculares foram diferentes entre os estudos.

Entretanto, este estudo apresenta uma temática de grande importância na avaliação das mulheres ao diagnóstico de câncer de mama: a relação entre a presença de comorbidade cardiovascular e a QVRS. Ambos os aspectos são preditores de pior mortalidade por câncer de mama $^{5,29}$ e devem ser incorporados como instrumentos na tomada de decisão terapêutica.

\section{CONCLUSÃO}

Ao diagnóstico de câncer de mama, 10,8\% das mulheres apresentavam problemas no sistema coração e $48,2 \%$ no sistema vascular. Observou-se maior risco de doenças no sistema coração nas mulheres com piores funçóes física e sexual, e naquelas com piores sintomas de dor e dispneia. Para o sistema vascular, verificou-se maior risco naquelas com piores funçôes física e sexual e pior satisfação sexual. Para sintomas na mama e perspectiva futura, aquelas com melhores escores apresentaram menor risco de problemas no sistema vascular.

\section{CONTRIBUIÇÕES}

Todos os autores contribuíram na concepção e planejamento do estudo; obtenção, análise e interpretação dos dados; redaçáo do manuscrito e aprovaram a versão final a ser publicada.

\section{DECLARAÇÃO DE CONFLITO DE INTERESSE}

A autora Anke Bergmann declara potencial conflito de interesses pela condição de ser a editora científica da Revista Brasileira de Cancerologia do INCA. Os demais autores não possuem conflito de interesses.

\section{FONTES DE FINANCIAMENTO}

Não há.

\section{REFERÊNCIAS}

1. Bray F, Ferlay J, Soerjomataram I, et. al. Global cancer statistics 2018: GLOBOCAN estimates of incidence and mortality worldwide for 36 cancers in 185 countries. CA Cancer J Clin. 2018;68(6):394-424. doi: https://doi. org/10.3322/caac. 21492

2. Instituto Nacional de Câncer José Alencar Gomes da Silva. Estimativa 2018: incidência de câncer no Brasil [Internet]. Rio de Janeiro: INCA; 2017. [acesso 2018 dez. 17]. Disponível em: http://www1.inca.gov.br/estimativa/2018/

3. Drope J, Schluger NW, editors. The tobacco atlas. 6th ed. Atlanta, GA: American Cancer Society; 2018.

4. World Health Organization. WHO methods for life expectancy and healthy life expectancy. Geneva: WHO; 2014.

5. Patnaik JL, Byers T, DiGuiseppi C, et al. Cardiovascular disease competes with breast cancer as the leading cause of death for older females diagnosed with breast cancer: a retrospective cohort study. Breast Cancer Res. 2011 Jun;13(3):R64. doi: https://doi.org/10.1186/bcr2901

6. Martel S, Maurer C, Lambertini M, et al. Breast cancer treatment-induced cardiotoxicity. Expert Opin Drug Saf. 2017 Sep;16(9):1021-38. doi: https://doi.org/10.1080/ 14740338.2017.1351541

7. Willson ML, Burke L, Ferguson T, et al. Taxanes for adjuvant treatment of early breast cancer. Cochrane Database Syst Rev. 2019 Sep 2;9:CD004421. doi: https://doi.org/10.1002/14651858.CD004421.pub3

8. Ewer SM, Pham DD. Late-onset heart failure after treatment for breast cancer. Cancer. [First published 2019 Aug 27]. doi: https://doi.org/10.1002/cncr.32483. 
9. Migowski A, Nadanovsky P, Vianna CMM. Mortalidade cardiovascular associada ao rastreamento mamográfico. Rev Bras Cancerol. 2019;65(3):e-02335. doi: https:// doi.org/10.32635/2176-9745.RBC.2018v65n3.335

10. Buscariollo DL, Cronin AM, Borstelmann NA, et al. Impact of pre-diagnosis depressive symptoms and health-related quality of life on treatment choice for ductal carcinoma in situ and stage I breast cancer in older women. Breast Cancer Res Treat. 2019 Feb;173(3):709-717. doi: https://doi.org/10.1007/ s10549-018-5006-5

11. Medina JMR, Trugilho IA, Mendes GNB, et al. Advanced clinical stage at diagnosis of breast cancer is associated with poorer health-related quality of life: a cross-sectional study. Eur J Breast Health. 2018 Dec 19;15(1):26-31. doi: https://doi.org/10.5152/ejbh.2018.4297

12. Karlsen RV, Frederiksen K, Larsen MB, et al. The impact of a breast cancer diagnosis on health-related quality of life. A prospective comparison among middle-aged to elderly women with and without breast cancer. Acta Oncol. 2016 Jun;55(6):720-7. doi: https://doi.org/10. 3109/0284186X.2015.1127415

13. Goyal NG, Levine BJ, Van Zee KJ, et al. Trajectories of quality of life following breast cancer diagnosis. Breast Cancer Res Treat. 2018 May;169(1):163-73. doi: https:// doi.org/10.1007/s10549-018-4677-2

14. Rey-Villar R, Pita-Fernández S, Cereijo-Garea C, et al. Quality of life and anxiety in women with breast cancer before and after treatment. Rev Latino-Am Enfermagem. 2017;25:e2958. doi: https://doi.org/10.1590/15188345.2258 .2958

15 - Organização Mundial da Saúde. CID -O: Classificação Internacional de Doenças para Oncologia. 3. ed. São Paulo: Edusp; 2005.

16. Fayers P, Bottomley A; EORTC Quality of Life Group; Quality of Life Unit. Quality of life research within the EORTC-the EORTC QLQ-C30. Eur J Cancer. 2002 Mar; 38(Suppl 4):S125-33. doi: https://doi.org/10.1016/ S0959-8049(01)00448-8

17. Miller MD, Paradis CF, Houck PR, et al. Rating chronic medical illness burden in geropsychiatric practice and research: application of the cumulative illness rating scale. Psychiatry Res. 1992;41(3):237-48. doi: https:// doi.org/10.1016/0165-1781(92)90005-n

18. Linn BS, Linn MW, Gurel L. Cumulative illness rating scale. J Am Geriatr Soc. 1968;16(5):622-6. doi: https:// doi.org/10.1111/j.1532-5415.1968.tb02103.x

19. Nabhan C, Smith SM, Helenoswski I, et al. Analysis of very elderly ( $\geq 80$ years) non-hodgkin lymphoma: impact of functional status and co-morbidities on outcome. $\mathrm{Br}$ J Haematol. 2012;156(2):196-204. doi: https://doi. org/10.1111/j.1365-2141.2011.08934.x

20. Puts MTE, Hardt J, Monette J, et al. Use of geriatric assessment for older adults in the oncology setting: a systematic review. J Natl Cancer Inst. 2012;104(15):113464. doi: https://doi.org/10.1093/jnci/djs285

21. Extermann M, Wedding U. Comorbidity and geriatric assessment for older patients with hematologic malignancies: a review of the evidence. J Geriatric Oncol. 2012;3(1):49-57. doi: https://doi.org/10.1016/j. jgo.2011.11.004

22. Ørum M, Jensen K, Gregersen M, et al. Impact of comprehensive geriatric assessment on short-term mortality in older patients with cancer-a follow-up study. Eur J Cancer. 2019;116:27-34. doi: https://doi. org/10.1016/j.ejca.2019.05.003

23. Organização Mundial da Saúde. CID -10: Classificação Estatística Internacional de Doenças e Problemas Relacionados à Saúde. São Paulo: Edusp; 2008.

24. Cardinale D, Caruso V, Cipolla CM. The breast cancer patient in the cardioncology unit. J Thorac Dis. 2018 Dec;10(Suppl 35):S4306-S4322. doi: https://doi. org/10.21037/jtd.2018.10.06

25. Nowsheen S, Duma N, Ruddy KJ. Preventing Today's Survivors of Breast Cancer From Becoming Tomorrow's Cardiac Patients. J Oncol Pract. 2018 Apr;14(4):213-4. doi: https://doi.org/10.1200/JOP.18.00130

26. Gulati M, Mulvagh SL. The connection between the breast and heart in a woman: breast cancer and cardiovascular disease. Clin Cardiol. 2018 Feb;41(2):253-7. doi: https:// doi.org/10.1002/clc.22886

27. Ng HS, Vitry A, Koczwara B, et al. Patterns of comorbidities in women with breast cancer: a Canadian population-based study. Cancer Causes Control. 2019 Sep;30(9):931-41. doi: https://doi.org/10.1007/s10552019-01203-0

28. Lorenz E, Blettner M, Lange B, et al. Prevalence of cardiac disease in breast cancer patients at time of diagnosis compared to the general female population in Germany. Breast Care (Basel). 2018 Aug;13(4):264-71. doi: https:// doi.org/10.1159/000487261

29. De Aguiar SS, Bergmann A, Mattos IE. Quality of life as a predictor of overall survival after breast cancer treatment. Qual Life Res. 2014 Mar;23(2):627-37. doi: https://doi. org/10.1007/s11136-013-0476-8 